\title{
TRIPLE SYSTEMS WITH A FIXED NUMBER OF REPEATED TRIPLES
}

\author{
C. A. RODGER \\ (Received 29 January 1984; revised May 14 1985) \\ Communicated by W. D. Wallis
}

\begin{abstract}
In this paper, linear embeddings of partial designs into designs are found where no repeated blocks are introduced in the embedding process. Triple systems, pure cyclic triple systems, cyclic and directed triple systems are considered. In particular, a partial triple system with no repeated triples is embedded linearly in a triple system with no repeated triples.
\end{abstract}

1980 Mathematics subject classification (Amer. Math. Soc.): 05 B 05.

\section{Introduction}

A block design $B[k, \lambda ; v]$ is a pair $(V, B)$ where $V$ is a set of $v$ symbols and $B$ is a collection of $k$-tuples called blocks such that each pair of symbols occurs together in precisely $\lambda$ blocks; if $k=3$, it is called a triple system, and if in addition $\lambda=1$, it is a Steiner triple system. A cyclic block design $C B[k, \lambda ; v]$ is a pair $(V, B)$, where each block contains the ordered pair $(x, y)$ if $x$ precedes and is adjacent to $y$ in the block, where the last symbol is assumed to precede and be adjacent to the first, and where each ordered pair occurs together in precisely $\lambda$ blocks; if $k=3$ and $\lambda=1$, it is called a Mendelsohn triple system. A directed block design $D B[k, \lambda ; v]$ is a pair $(V, B)$, where a block in $B$ contains the ordered pair $(x, y)$ if $x$ precedes $y$, and where each ordered pair occurs together in precisely $\lambda$ blocks. Partial designs result if the above definitions are relaxed to allow each pair (ordered pair) to occur together in at most $\lambda$ blocks.

(C) 1986 Australian Mathematical Society $0263-6115 / 86 \$ A 2.00+0.00$ 
The problem of embedding partial Steiner triple systems $\left(P, p_{1}\right)$ and $\left(P, p_{2}\right)$ in Steiner triple systems $\left(S, s_{1}\right)$ and $\left(S, s_{2}\right)$, respectively, so that $s_{1} \cap s_{2}=p_{1} \cap p_{2}$ was considered by Hall and Udding [6] and by Lindner [10]. This problem was generalized by Lindner [10], who embedded a collection of $k$ partial Steiner triple systems on $n$ symbols in an intersection preserving set of $k$ Steiner triple systems, each on $v$ symbols, for every $v \geqslant 2 k(4 n+1), v \equiv 1$ or $3(\bmod 6)$. Lindner, Poucher and Rosa have also considered the intersection preserving finite embeddability property for other designs (see, for example, [11], [12], [13]).

In this paper we consider a related problem where a partial triple system of order $n$ is embedded in a triple system of order approximately $9 \lambda^{2} n$ (see Theorem 3 ), and where no repeated blocks are introduced in the embedding procedure (the smallest known embedding of partial triple systems (with no restrictions) is at present approximately $6 \lambda n$ (see [2])). An obvious corollary is that a partial triple system with no repeated blocks can be embedded linearly in a triple system with no repeated blocks.

A cyclic triple system is pure if no block occurs more than once in $B$, and if $\{i, j, k\} \in B$ implies that $\{i, k, j\} \notin B$ (so the underlying triple system has no repeated blocks). Pure cyclic triple systems were introduced by di Paola and Nemeth [5]. Here we show that a partial pure cyclic triple system can be linearly embedded in a pure cyclic triple system.

Finally, linear embeddings of partial cyclic and partial directed triple systems are found in which no repeated cyclic and directed triples, respectively, are added during the embedding process.

A quasigroup $(Q, \cdot)$ of order $v$ is symmetric if for all $i, j$ with $1 \leqslant i \leqslant j \leqslant v$, we have $i \cdot j=j \cdot i$; it is antisymmetric if for all $i, j$ with $1 \leqslant i<j \leqslant v$, we have $i \cdot j \neq j \cdot i$; and it is idempotent if for all $i$ with $1 \leqslant i \leqslant v$, we have $i \cdot i=i$. Two partial triple systems $P$ and $Q$ are said to be mutually balanced when, for each pair of symbols $x$ and $y, x$ and $y$ occur together in $z$ blocks of $P$ if and only if $x$ and $y$ occur together in $z$ blocks of $Q$.

In this paper, in counting triples we include repeated triples as many times as they occur. Also $[x]$ denotes the greatest integer less than or equal to $x$.

\section{Triple systems}

The embedding techniques used here are a variation on the technique developed in [2], [3], [7] and [8], from which the following can easily be obtained.

LeMma 1. Let $S$ be a partial triple system $B[3, \lambda ; n]$ with a repeated triples (Let $S$ be a partial pure cyclic triple system $C B[3, \lambda ; n]$.$) Then there exist two partial$ 
triple systems (partial pure cyclic triple systems) of order $t$ and index $\lambda$, say $T$ and $T^{\prime}$, where $t \leqslant(3 \lambda+2) n / 2(t \leqslant(3 \lambda+1) n)$, and where

(1) $S$ is embedded in $T$;

(2) $T$ and $T^{\prime}$ are mutually balanced;

(3) $T^{\prime}$ contains no repeated triples ( $T^{\prime}$ is pure), and $T^{\prime}$ can be decomposed into $\lambda$ partial Steiner triple systems ( $\lambda$ partial pure Mendelsohn triple systems); and

(4) the only repeated triples in $T$ are those in $S$ ( $T$ is pure).

To prove the main result we shall also use the following theorem.

THEOREM 2 [4]. A partial idempotent symmetric quasigroup of order $t$ can be embedded in an idempotent symmetric quasigroup of order $2 v+1$ for any $v \geqslant t$.

Theorem 3. A partial $B[3, \lambda ; n]$ with a repeated triples can be embedded in a $B[3, \lambda ; v]$ with a repeated triples, where

$$
v \leqslant 3(2[\lambda / 2]+1)((3 \lambda+2) n+1) .
$$

Proof. Let $S$ be a partial triple system with $\alpha$ repeated triples. From Lemma 1, let $T$ and $T^{\prime}$ be partial systems of order $t$ that satisfy conditions (1), (2), (3) and (4), where $t \leqslant(3 \lambda+2) n / 2$. Let $T^{\prime}$ be decomposed into partial Steiner triple systems $V_{1}, \ldots, V_{\lambda}$.

For each $x, 1 \leqslant x \leqslant \lambda$, define a partial symmetric idempotent quasigroup of order of $t$ with product ${ }_{x}$ such that for each triple $\{i, j, k\}$ in $V_{x}$, we have $i \cdot{ }_{x} j=k, j \cdot{ }_{x} k=i$, and $k \cdot{ }_{x} i=j$; then, using Theorem 2, embed this in an idempotent symmetric quasigroup $\left(Q_{x},{ }^{\cdot}\right)$ of order $2 t+1$.

For each $x, 1 \leqslant x \leqslant \lambda$, form the quasigroups $\left(P_{1, x}, \odot_{(1, x)}\right)$ and $\left(P_{2, x}, \odot_{(2, x)}\right)$, each of order $2[\lambda / 2]+1$, with $P_{1, x}=P_{2, x}=\{0,1, \ldots, 2[\lambda / 2]\}$, by defining

$$
i \odot_{(1, x)} j=\left(\left[\frac{\lambda}{2}\right]+1\right)(i+j)+(x-1)
$$

and

$$
i \odot_{(2, x)} j=\left(\left[\frac{\lambda}{2}\right]+1\right)(i+j)+(x-1)\left[\frac{\lambda}{2}\right],
$$

for $0 \leqslant i, j \leqslant 2[\lambda / 2]$, reducing symbols modulo $2[\lambda / 2]+1$. Then clearly $P_{1,1}, \ldots, P_{1, \lambda}$ form a collection of pairwise disjoint symmetric diagonal quasigroups. Also, since $[\lambda / 2]$ and $2[\lambda / 2]+1$ are relatively prime, $P_{2,1}, \ldots, P_{2, \lambda}$ form a collection of pairwise disjoint symmetric diagonal quasigroups.

It will be relevant later to note that for each $x, 1 \leqslant x \leqslant \lambda$, and for each $j$, $0 \leqslant j \leqslant 2[\lambda / 2]$, we have

$$
\begin{gathered}
j \odot_{(1, x)} j \equiv j+x-1, \\
\omega=\left(j \odot_{(1, x)} j\right) \odot_{(2, x)}\left(j \odot_{(1, x)} j\right) \equiv j+(x-1)\left[\frac{\lambda+2}{2}\right],
\end{gathered}
$$


and so

$$
\omega \bigodot_{(2, x)} \omega \equiv j,
$$

where all congruences are modulo $2[\lambda / 2]+1$.

Let $u=(2[\lambda / 2]+1)(2 t+1)$. For each $x, 1 \leqslant x \leqslant \lambda$, form the quasigroup $\left(R_{i, x},{ }^{\cdot}(i, x)\right)$, where $1 \leqslant i \leqslant 2$, of order $u$, with $R_{i, x}=\{1,2, \ldots, u\}$, by the "direct product" of $Q_{x}$ and $P_{i, x}$; thus, for each $l, j$ in $P_{i, x}$, if $l \odot_{(i, x)} j=k$, then replace $k$ with a copy of $Q_{x}+k(2 t+1)$ (that is, add $k(2 t+1)$ to each entry in $\left.Q_{x}\right)$. Then $R_{i, 1}, \ldots, R_{i, \lambda}$ form a collection of pairwise disjoint symmetric quasigroups, for $i=1$ or 2 .

For each $x, 1 \leqslant x \leqslant \lambda$, form the Steiner triple system $\left(S_{x}, t_{x}\right)$ on the symbols $(i, j)$, for $1 \leqslant i \leqslant 3$ and $1 \leqslant j \leqslant u$, as follows:

(i) for each $j, 1 \leqslant j \leqslant u$,

$$
\left\{(1, j),\left(2, j \cdot{ }_{(1, x)} j\right),\left(3,\left(j \cdot_{(1, x)} j\right) \cdot_{(2, x)}\left(j \cdot{ }_{(1, x)} j\right)\right)\right\} \in t_{x} ;
$$

(ii) for each $j, k$ with $1 \leqslant j<k \leqslant u, t_{x}$ contains

$$
\{(1, j),(1, k),(2, j \cdot(1, x) k)\},\left\{(2, j),(2, k),\left(3, j \cdot{ }_{(2, x)} k\right)\right\}
$$

and $\{(3, j),(3, k),(1, j \cdot(2, x) k)\}$.

Then, since $R_{i, 1}, \ldots, R_{i, \lambda}$ are pairwise disjoint for $i=1$ or 2 , no triple occurs in more than one of $S_{1}, \ldots, S_{\lambda}$.

If $(a, b, c)$ is a triple in $V_{x}$, then let $A=b \cdot{ }_{(1, x)} c, B=a \cdot{ }_{(1, x)} c, C=a \cdot{ }_{(1, x)} b$, $\alpha=B \cdot{ }_{(2, x)} C, \beta=A \cdot{ }_{(2, x)} C$ and $\gamma=A \cdot_{(2, x)} B$. Then $S_{x}$ contains a triple system on the nine points $(1, a),(1, b),(1, c),(2, A),(2, B),(2, C),(3, \alpha),(3, \beta)$ and $(3, \gamma)$; this can be seen from the construction of $S_{x}$ together with equations $(*)$. For each triple in $V_{x}$, say $(a, b, c)$, unplug this subsystem on nine points and replace it with a subsystem that contains the triples $\{(1, a),(1, b),(1, c)\},\{(2, A)$, $(2, B),(2, C)\},\{(3, \alpha),(3, \beta),(3, \gamma)\},\{(1, a),(2, A),(3, \alpha)\},\{(1, b),(2, B),(3, \beta)\}$, and $\{(1, c),(2, C),(3, \gamma)\}$ to form the Steiner triple system $\left(S_{x}^{\prime}, t_{x}^{\prime}\right)$. Then no triple occurs in more than one of $S_{1}^{\prime}, \ldots, S_{\lambda}^{\prime}$. Also, $S_{x}^{\prime}$ contains a copy of $V_{x}$, since if $\{a, b, c\}$ is a triple in $V_{x}$, then $\{(1, a),(1, b),(1, c)\} \in t_{x}^{\prime}$.

Combine $S_{1}^{\prime}, \ldots, S_{\lambda}^{\prime}$ to form a $B[3, \lambda ; u]$, say $W$, which has no repeated triples, and which has a copy of $T^{\prime}$ on the points $(1, j)$ for $1 \leqslant j \leqslant t$. Since the only triples in $W$ in which the three points all have first coordinate 1 are those in $T^{\prime}$, replacing triples in $T^{\prime}$ by those in $T$ creates no repeated triples except for those in $T$. This produces the required embedding.

\section{Pure cyclic triple systems}

We shall need the following lemmas. The first is proved using an adaption of Hilton's method [9] for embedding partial idempotent latin squares. 
LeMma 4. A partial antisymmetric idempotent latin square of order $n$ can be embedded in an antisymmetric idempotent latin square of order $4 n$.

Proof. Let $P$ be a partial antisymmetric idempotent latin square of order $n$. Fill the empty cells of $P$ using symbols $1, \ldots, 2 n$ to produce an incomplete antisymmetric idempotent latin square of order $n$, call it $A_{1}$, and embed $A_{1}$ in a latin square $A$ of order $2 n$. Subdivide $A$ as in Figure 1, with $x=A$.

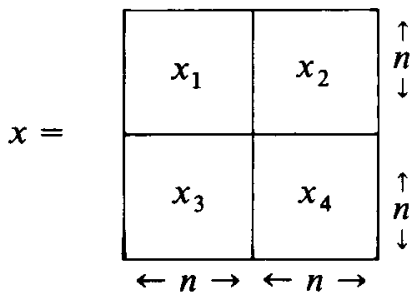

Figure 1

Form a latin square $B$ by adding $n$ to each entry at $A^{T}$, reducing entries modulo $2 n$. Form a latin square $C$ by adding $2 n$ to each entry in $A$, and form a latin square $D$ by adding $2 n$ to each entry in $B$. Subdivide $B, C$ and $D$ as depicted in Figure 1. Then the required embedding of $P$ can be formed as indicated in Figure 2.

\begin{tabular}{|c|c|c|c|}
\hline 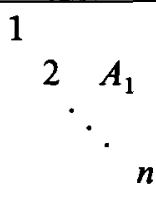 & $D_{4}$ & $A_{2}$ & $D_{3}$ \\
\hline$C_{4}$ & $\begin{array}{c}n+1 \\
B_{1} \\
\ddots \\
\\
2 n\end{array}$ & $C_{3}$ & $B_{2}$ \\
\hline$C_{2}$ & $B_{3}$ & $\begin{array}{cc}2 n+1 & \\
C_{1} & \\
\ddots & \\
& 3 n\end{array}$ & $B_{4}$ \\
\hline$A_{3}$ & $D_{2}$ & $A_{4}$ & $\begin{array}{c}3 n+1 \\
D_{1} \\
\ddots \\
\\
\end{array}$ \\
\hline
\end{tabular}

Figure 2. The required embedding of $P$ 
THeOREM 5.AA partial pure $C B[3, \lambda ; n]$ can be embedded in a pure $C B[3, \lambda ; v]$, where

$$
v \leqslant 3(2[\lambda / 2]+1)(12 \lambda+4) n+1 .
$$

Proof. The proof closely follows that of Theorem 3, so we describe only the main differences.

Let $S$ be a partial pure cyclic triple system of order $n$. Lemma 1 is used to construct partial pure cyclic triple systems $T$ and $T^{\prime}$, where $T^{\prime}$ is decomposed into $\lambda$ partial pure Mendelsohn triple systems $V_{1}, \ldots, V_{\lambda}$. For each $x, 1 \leqslant x \leqslant \lambda$, a partial idempotent antisymmetric quasigroup corresponding to $V_{x}$ is formed which is embedded in an idempotent antisymmetric quasigroup $Q_{x}$ of order $4 t$ using Lemma 4.

Construct the collection of pairwise disjoint symmetric quasigroups $P_{i, 1}, \ldots, P_{i, \lambda}$, for $i=1$ and 2, as in Theorem 3.

Let $u=(2[\lambda / 2]+1) 4 t$. Let $Q_{x}^{\prime}$ be the antisymmetric quasigroup of order $4 t$ formed by adding 1 to each entry in $Q_{x}^{T}$, reducing the symbol modulo $4 t$. For each $x, 1 \leqslant x \leqslant \lambda$, form the quasigroup $\left(R_{i, x}, \cdot_{(i, x)}\right)$, where $1 \leqslant i \leqslant 2$, of order $u$, by a type of direct product as follows: for each $l, j$ such that $1 \leqslant l$, $j \leqslant 2[\lambda / 2]+1$, if $\bigodot_{(i, x)} j=k$, then replace $k$ with a copy of $Q_{x}+4 t k$ when $l \geqslant j$, and with a copy of $Q_{x}^{\prime}+4 t k$ when $l<j$. Then $R_{i, 1}, \ldots, R_{i, \lambda}$, for $i=1$ and 2 , are pairwise disjoint and are antisymmetric.

Construct $\lambda$ pure Mendelsohn triple systems $\left(S_{x}, t_{x}\right)$ on the set of symbols $\{\infty\} \cup\{(i, j) \mid 1 \leqslant i \leqslant 3$ and $1 \leqslant j \leqslant u\}$ as follows:

(a) for each $j, 1 \leqslant j \leqslant u, t_{x}$ contains

$$
\begin{aligned}
& \left\{\infty,(1, j),\left(2, j \cdot{ }_{(1, x)} j\right)\right\}, \\
& \left\{\infty,\left(2, j \cdot \cdot_{(1, x)} j\right),\left(3,\left(j \cdot{ }_{(1, x)} j\right) \cdot{ }_{(2, x)}\left(j \cdot{ }_{(1, x)} j\right)\right)\right\}, \\
& \left\{\infty,\left(3,\left(j \cdot \cdot_{(1, x)} j\right) \cdot \cdot_{(2, x)}\left(j \cdot \cdot_{(1, x)} j\right)\right),(1, j)\right\}, \\
& \left\{(1, j),\left(3,\left(j \cdot \cdot_{(1, x)} j\right) \cdot \cdot_{(2, x)}\left(j \cdot \cdot_{(1, x)} j\right)\right),\left(2, j \cdot_{(1, x)} j\right)\right\} ;
\end{aligned}
$$

(b) for each $j$ and $k$ with $1 \leqslant j, k \leqslant u, t_{x}$ contains $\left\{(1, j),(1, k),\left(2, j \cdot \cdot_{(1, x)} k\right)\right\}$, $\left\{(2, j),(2, k),\left(3, j \cdot{ }_{(2, x)} k\right)\right\}$, and $\left\{(3, j),(3, k),\left(1, j \cdot{ }_{(2, x)} k\right)\right\}$.

The proof now follows that of Theorem 3 , and the details are omitted.

\section{Other results}

We can obtain results similar to Theorem 3 for cyclic triple systems and for directed triple systems.

THEOREM 6 [1]. A partial idempotent latin square of order $n$ can be embedded in an idempotent latin square of order $2 n+1$. 
THEOREM 7. A partial $C B[3, \lambda ; n]$ with $\alpha$ repeated cyclic triples can be embedded in a $C B[3, \lambda ; v]$ with $\alpha$ repeated triples, where

$$
v \leqslant 3(2[\lambda / 2]+1)((3 \lambda+2) n+1) .
$$

Proof. The proof closely follows that of Theorem 3, except that Theorem 6 is used instead of Theorem 2 . The details are omitted.

THEOREM 8. A partial $D B[3, \lambda ; n]$ with $\alpha$ repeated directed triples can be embedded in a $D B[3, \lambda ; v]$ with a repeated directed triples, where

$$
v \leqslant 3(2[\lambda / 2]+1)((6 \lambda+2) n+1) .
$$

Proof. Lemma 1 can be stated for directed triple systems, where $t \leqslant(3 \lambda+1) n$, where $T$ is a partial directed triple system containing $S$, and where $T^{\prime}$ is a partial cyclic triple system with no repeated cyclic triples (see [3]). The proof closely follows that of Theorem 3 using the embedding technique developed in [2] and [3].

\section{Conclusion}

The same techniques that are described here can be used to embed partial triple systems $\left(P_{1}, p_{1}\right), \ldots,\left(P_{k}, p_{k}\right)$ into triple systems $\left(S_{1}, s_{1}\right), \ldots,\left(S_{k}, s_{k}\right)$, respectively, where for $1 \leqslant i<j \leqslant k$, where $p_{i} \cap p_{j}=s_{i} \cap s_{j}$, and they may be applied to analogous problems for cyclic and directed triple systems. The size of the embedding increases by a factor of approximately $2[k / 2]+1$.

\section{References}

[1] L. D. Andersen, A. J. W. Hilton and C. A. Rodger, 'A solution to the embedding problem for partial idempotent latin squares', J. London Math. Soc. 26 (1982), 21-27.

[2] C. J. Colbourn, R. C. Hamm, C. C. Lindner and C. A. Rodger, 'Embedding partial graph designs, block designs and triple systems with $\lambda>1$ ', Canad. Math. Bull., to appear.

[3] C. J. Colbourn, R. C. Hamm and C. A. Rodger, 'Small embeddings of partial directed triple systems and partial triple systems with even $\lambda$ ', J. Combin. Theory Ser. A 37 (1984), 363-369.

[4] A. Cruse, 'On embedding incomplete symmetric latin squares', J. Combin. Theory Ser. A 16 (1974), 18-27.

[5] J. W. diPaola and E. Nemeth, 'Generalized triple systems and medial quasigroups', Proc. 7th Southeastern Conf. on Combinatorics, Graph Theory and Computing, 1976, 289-306.

[6] J. I. Hall and J. T. Udding, 'On intersection pairs of Steiner Triple Systems', Indag Math. 39 (177), 87-100 (= Proc. Konikl. Nederl. Akad. Wetensch. Ser. A 80 (1977)). 
[7] R. C. Hamm, 'Embedding partial transitive triple systems', Proc. 14th Southeastern Conf. on Combinatorics, Graph Theory and Computing, 1983, 447-453.

[8] R. C. Hamm, C. C. Lindner and C. A. Rodger, 'Linear embeddings of partial directed triple systems with $\lambda=1$ and partial triple systems with $\lambda=2$ ', Ars Combin. 16 (1983), 11-16.

[9] A. J. W. Hilton, 'Embedding an incomplete diagonal latin square in a complete diagonal latin square', J. Combin. Theory Ser. A 15 (1973), 121-128.

[10] C. C. Lindner, 'A survey of embedding theorems for Steiner systems', Ann. Discrete Math. 7 (1980), 175-202.

[11] C. C. Lindner and T. Evans, 'Finite embedding theorems for partial designs and algebras', Séminaires de Mathématiques Supérieures, 56, Les Presses de Accents l'Université de Montréal, Montreal, 1977.

[12] C. C. Lindner and A. Rosa, 'Finite embedding theorems for partial Steiner triple systems', Discrete Math. 13 (1975), 31-39.

[13] W. B. Poucher, 'Finite embedding theorems for partial pairwise balanced designs', Discrete Math. 18 (1977), 291-298.

Department of Mathematics

Auburn University

Auburn, Alabama 36849

U.S.A. 